\title{
Disturbance of SH-type waves due to moving stress discontinuity in an anisotropic soil layer overlying an inhomogeneous elastic half-space
}

\author{
D MANDAL ${ }^{1,2 *}, \mathrm{PC} \mathrm{PAL}^{2}$ and $\mathrm{S} \mathrm{KUMAR}^{2}$ \\ ${ }^{1}$ Government Engineering College, Chaibasa 833215, India \\ ${ }^{2}$ Department of Applied Mathematics, Indian School of Mines, \\ Dhanbad 826 004, India \\ e-mail: dinbandhumandal@gmail.com
}

MS received 1 December 2012; revised 6 September 2013; accepted 23 September 2013

\begin{abstract}
The disturbance and propagation of SH-type waves in an anisotropic soil layer overlying an inhomogeneous elastic half-space by a moving stress discontinuity is considered. Stress discontinuity moves with non-uniform velocity and is impulsive in nature. The displacements are obtained in exact form by the method due to Cagniard modified by de Hoop. The numerical result is calculated for special cases and the natures are depicted graphically.
\end{abstract}

Keywords. SH-type waves; anisotropic soil layer; inhomogeneous media; Cagniard-de Hoop technique; moving stress discontinuity.

\section{Introduction}

The two main types of seismic waves are body waves and surface waves. The first kind of body waves is the $\mathrm{P}$-wave or primary waves. The second type of body wave is the S-wave or secondary waves which have a transverse, shear vibration in a plane perpendicular to the direction of propagation. Two polarizations of shear waves are possible (i) one in vertical direction named as $\mathrm{SV}$-waves, (ii) another one in horizontal direction named as SH-waves. The anisotropy present in rock types of crust would affect the speed of propagation of SV-waves as well as SH-waves. Individual grains of most solids are highly anisotropic, direction and position conscious. Thus, anisotropic and inhomogeneity affect the speed of propagation of SH-types waves.

While both soil and rocks are concerned with pores, there is a considerable difference in the nature of the medium: soil are usually regarded as consisting of discrete particles touching at isolated points, while materials such as sedimentary rocks and concrete are regarded as a solid skeleton traversed by a fine network of capillaries. Some soils behave as anisotropic materials where swelling of some constituents on wetting, is ignored.

*For correspondence 
Surface wave propagation involves transition layers which in general are both anisotropic and heterogeneous. The effect of anisotropy on reflection and transmission of elastic waves at a plane interface between two elastic media was studied by Daley \& Hron (1977). Brock (1982) has considered the effect of displacement discontinuity derivatives on wave propagation prescribed along arbitrarily time-varying line segments normal to an elastic half-plane surface or bimaterial interface. Nag \& Pal (1977) have considered the disturbance of SH-type waves due to shearing stress discontinuity in an elastic layered half-space and considered two types of shearing stress discontinuity for finding the displacement at the free surface. Pal \& Debnath (1979) have studied the Generation of SH-type waves at the free surface of a layered anisotropic elastic media due to an impulsive stress discontinuity moving with uniform velocity along the interface of the layered medium.

The generation of SH-type wave due to non-uniformly moving stress discontinuity in a layered half space has been considered by Mittal \& Sidhu (1982). Romeo (1997) has investigated the SH surface waves on a layered anisotropic half-space is having recourse to a wave-splitting approach. The displacements have been evaluated using de Hoop's version of Cagniard's technique. Sengupta \& Nath (2001) have investigated the surface waves in fibre-reinforced anisotropic elastic media. de Hoop (2002) has considered the reflection and transmission properties of an elastic interfacial bonding of two semi-infinite solids and are investigated for the simplest possible case of a line-source excited two-dimensional SH-wave. Tomar \& Kaur (2007) have studied the reflection and transmission of a plane SH-wave incident at a corrugated interface between a dry sandy half-space and an anisotropic elastic half-space. All the above authors have not considered the effect of shearing stress discontinuity for the propagation of $\mathrm{SH}$-waves with uniform or non-uniform velocity in sandy materials.

Shearing stress discontinuity occurs in many cases e.g.,: (i) inside the earth between two layers, if there is a crack which is being filled up by liquid, (ii) when a layer tends to slide or slip without friction over another layer inside the earth. For the above reasons, the stress discontinuity may be treated as a moving source either with uniform velocity or with non-uniform velocity. Relating to stress discontinuity, Pal (1983) has considered the problem of generation of SH-type waves due to non-uniformly moving stress discontinuity in a layered anisotropic elastic half-space. It is our intention in the present paper, to investigate the disturbance of SH-type waves at the free surface of a anisotropic soil layer due to an impulsive stress discontinuity moving with nonuniform velocity along the interface of isotropic homogeneous and inhomogeneous medium. The displacement is calculated numerically at a particular distance on the surface for different types of the discontinuity in the shearing stress for different value of inhomogeneous parameter. It involves Laplace and Fourier transform and the inversion is based on Garvin's (1956) modification of Cagniard's (1962) method. The problem discussed may be of importance in connection with the propagation of cracks in layers and the numerical results are shown graphically.

\section{Formulation of the problem}

Let us consider an anisotropic soil layer I of thickness $\mathrm{h}$ with Weiskopf anisotropy which is lying over an inhomogeneous elastic half-space (layer II) with shear modulus $\mu_{2}=\mu_{0} e^{b z}$ and density $\rho_{2}=\rho_{0} e^{b z}$. The problem is two-dimensional and is being analysed in $x z$-plane. The positive $z$-axis is directed vertically downwards and $x$-axis is along the interface $z=0$. Here, we consider the transient wave motion due to a shearing stress discontinuity which is being created at a certain instant at the point $(x, 0)$ and then moves along $x$-axis with time dependent speed (figure 1). 
For SH-type wave which is transverse in horizontal plane propagating along $\mathrm{x}$-axis, we have $u=w=0$ and $v=v(x, z, t)$ where $u, v, w$ are the displacement components along $x, y, z$ directions, respectively and independent of $y$-axis. As we are interested to calculate the transverse displacement $v_{1}$ of an element, so the equation of motion in anisotropic layer is (vide Pal 1983)

$$
N_{1} \frac{\partial^{2} v_{1}}{\partial x^{2}}+G_{1} \frac{\partial^{2} v_{1}}{\partial z^{2}}=\rho_{1} \frac{\partial^{2} v_{1}}{\partial t^{2}},
$$

where $N_{1}, G_{1}$ are the anisotropic parameter for soil and $\rho_{1}$ is the mass density of the soil.

For layer II the equation of motion for the transverse displacement $v_{2}$ is

$$
\frac{\partial^{2} v_{2}}{\partial x^{2}}+\frac{\partial^{2} v_{2}}{\partial z^{2}}+b \frac{\partial v_{2}}{\partial z}=\frac{1}{\beta_{2}^{2}} \frac{\partial^{2} v_{2}}{\partial t^{2}},
$$

where $\beta_{2}^{2}=\frac{\mu_{0}}{\rho_{0}}$ and $b$ is inhomogeneity parameter.

The boundary conditions are, in the usual notations,

$$
\begin{aligned}
\left(\tau_{y z}\right)_{1} & =0 \text { at } z=-h, \quad t>0 \quad \text { i.e., shearing stress vanishes at the free surface } z=-h v_{1} \\
& =v_{2} \text { at } z=0, \quad t>0 \quad \text { i.e., displacement are continuous at the interface } \\
\left(\tau_{y z}\right)_{1} & -\left(\tau_{y z}\right)_{2}=f(x, t) H(t) \quad \text { at } z=0
\end{aligned}
$$

where $f(x, t)$ will be chosen later on, and $H(t)$ is Heaviside's unit function of time $t$. This definition shows that the stress discontinuity at interface $z=0$ moves with non-uniform velocity. For two dissimilar layer stress discontinuity in shearing stress occurs at the interface where as the normal stress is continuous there. Since normal stress are continuous and these are related to normal strains with displacement components, so displacements are also continuous at $z=0$. When discontinuity moves naturally the displacement components in transverse directions also travels (or moves) with certain velocity.

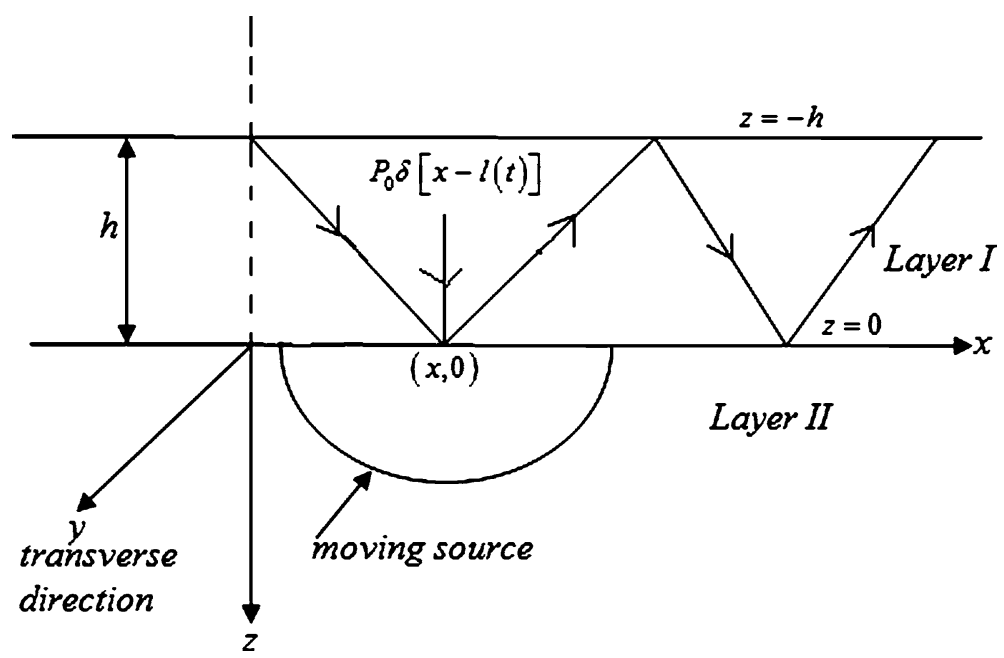

Figure 1. Geometry of problem. 


\section{Solution of problem}

The problem can readily be solved by using the Laplace and Fourier transforms combined with the modified Cagniard-de Hoop (1960) technique and used by Pal (1983). The Laplace transform of a function $v(x, z, t)$ with respect to $t$ will be defined by

$$
L[v(x, z, t)]=V(x, z, p)=\int_{-\infty}^{\infty} e^{-p t} v(x, z, t) d t,
$$

where $p$ is real and positive.

The Fourier transform $V(x, z, p)$ with respect to $\mathrm{x}$ will be written as

$$
\begin{gathered}
\bar{V}(\xi, z, p)=\int_{-\infty}^{\infty} e^{i \xi x} v(x, z, p) d x \\
V(x, z, p)=\frac{1}{2 \pi} \int_{-\infty}^{\infty} e^{-i \xi x} \bar{V}(\xi, z, p) d \xi .
\end{gathered}
$$

Now applying Laplace transform and Fourier transform to the equations of motion, we get solutions in the upper and lower layers with $V_{2} \rightarrow 0$ as $z \rightarrow \infty$ in the form:

$$
V_{1}=A \cosh a_{1} z+B \sinh a_{1} z \quad \text { at } \quad-h \leq z \leq 0,
$$

$$
V_{2}=C e^{-a_{2} z}
$$

where

$$
a_{1}=\left(\xi^{2} \phi_{1}^{2}+\frac{p^{2}}{\beta_{1}^{2}}\right)^{\frac{1}{2}}, \quad a_{2}=\frac{b+\sqrt{b^{2}+4\left(\xi^{2}+\frac{p^{2}}{\beta_{2}^{2}}\right)}}{2}, \quad \beta_{1}^{2}=\frac{G_{1}}{\rho_{1}}, \quad \phi_{1}^{2}=\frac{N_{1}}{G_{1}},
$$

where $\beta_{1}$ and $\beta_{2}$ are shear wave velocities in anisotropic soil layer and isotropic and inhomogeneous medium, $\phi_{1}$ is square root of ratio of anisotropic soil constant.

Taking the Laplace transform of boundary condition, we get

$$
\begin{gathered}
V_{1}=V_{2} \quad \text { at } z=0, \\
G_{1} \frac{d V_{1}}{d z}=0 \quad \text { at } z=-\mathrm{h}, \\
G_{1} \frac{d V_{1}}{d z}-\mu_{0} \frac{d V_{2}}{d z}=F \quad \text { at } \quad z=0 .
\end{gathered}
$$

Solving for A, B, C with the aid of equation (9), we have the displacement function at the free surface $z=-h$ given by

$$
V_{1}(x,-h, p)=\frac{1}{\pi} \int_{-\infty}^{\infty} \frac{\bar{F} e^{-a_{1} h} e^{-i \xi x}\left(1-K e^{-2 a_{1} h}\right)^{-1}}{G_{1} a_{1}+\mu_{0} a_{2}} d \xi,
$$


where

$$
K=\frac{G_{1} a_{1}-\mu_{0} a_{2}}{G_{1} a_{1}+\mu_{0} a_{2}} .
$$

As $\xi$ is complex, in order to apply Cagniard-de Hoop technique, we put $\xi=i k p$ and $b=p \gamma$ (as $b$ is dimension of length and so it is always $+\mathrm{ve}$ ), we find $a_{1}=b_{1} p, a_{2}=b_{2} p$, where

$$
b_{1}=\left(\frac{1}{\beta_{1}^{2}}-k^{2} \phi_{1}^{2}\right)^{\frac{1}{2}}, \quad b_{2}=\frac{\gamma+\sqrt{\gamma^{2}+4\left(\frac{1}{\beta_{2}^{2}}-k^{2}\right)}}{2} .
$$

Hence relation (10) can be written as

$$
V_{1}(x,-h, p)=\frac{i}{\pi} \int_{-\infty}^{\infty} \frac{\bar{F} e^{-p h b_{1}+k p x}\left(1-K e^{-2 p h b_{1}}\right)^{-1}}{G_{1} b_{1}+\mu_{0} b_{2}} d k,
$$

where

$$
K=\frac{G_{1} b_{1}-\mu_{0} b_{2}}{G_{1} b_{1}+\mu_{0} b_{2}},
$$

represents the reflection coefficient for $\mathrm{SH}$ waves incident from the anisotropic soil layer at the interface between the two media.

\section{Concentrated non-uniformly moving stress discontinuity}

Let us now define the unknown function $f(x, t)$ as considered by Freund (1972) and is given by

$$
f(x, t)=P_{0} \delta[x-l(t)],
$$

where $P_{0}$ is a constant and $\delta()$ is Dirac's delta function. Here $l(t)$ will be assumed to be a continuous, monotonically increasing function of time, i.e., it will never be acted at a single point for finite time. Function $l(t)$ is invertible, i.e., there exists a continuous and monotonically increasing function $t=\eta(x)$ such that

$$
l[\eta(x)]=x, \eta[l(t)]=t .
$$

Moreover, these function satisfy the following relations identically in $x$ or $t$ (after differentiation)

$$
\dot{l}[\eta(x)] \eta^{\prime}(x)=1, \quad \eta^{\prime}[l(t)] \dot{l}(t)=1,
$$

where $l^{\prime}(t)=d l / d t$ is the speed of propagation of stress discontinuity at any time $t$ and $\eta^{\prime}(x)=$ $\frac{d \eta}{d x}$ is related to slowness of stress discontinuity at any place $x$.

Since $l(0)=0$, assumption (12) shows that shearing stress discontinuity is at rest until time $t=0$, then begins to move according to $l(t)$ in positive $x$-direction.

Now applying Laplace transform to (12) we get

$$
F(x, t)=\int_{0}^{\infty} P_{0} \delta[x-l(t)] e^{-p t} d t
$$


but $\delta[x-l(t)]=\eta^{\prime}(x) \delta[t-\eta(x)]$. Hence

$$
F=\int_{0}^{\infty} P_{0} \eta^{\prime}(x) \delta[t-\eta(x)] e^{-p t} d t=P_{0} \eta^{\prime}(x) e^{-p \eta(x)} H[\eta(x)] .
$$

Taking Fourier transform of relation (14) we get

$$
\bar{F}=P_{0} \int_{0}^{\infty} \eta^{\prime}(x) e^{-p \eta(x)} H[\eta(x)] e^{-i \xi x} d x
$$

Putting $\xi=i k p$,

$$
\bar{F}=P_{0} \int_{0}^{\infty} \eta^{\prime}(s) e^{-p \eta(s)} e^{k p s} d s,
$$

where, we have changed the variable of integration from $x$ to $s$ and made use of the following results:

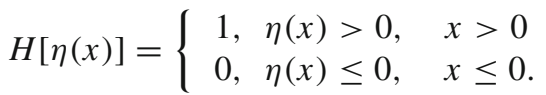

\section{Solid displacement on the free surface $(z=-h)$}

From equations (11) and (16), we have

$$
V_{1}(x,-h, p)=\frac{i P_{0}}{\pi} \int_{0}^{\infty} \eta^{\prime}(s) e^{-p \eta(s)} \int_{-\infty}^{\infty} \frac{e^{-p\left(b_{1} h-k x+k s\right)}}{G_{1} b_{1}+\mu_{0} b_{2}}\left[1+K e^{-2 p b_{1} h}+K^{2} e^{-4 p b_{1} h}+\ldots .\right] d k d s,
$$

where $K$ is defined earlier in equation (11). The coefficients of different powers of $K$ in the series of equation (18) are associated with pulses undergoing repeated reflections in the upper layer.

Now Laplace inversion of $V_{1}(x,-h, p)$ can be done by Cagniard's technique modified by de Hoop (1960).

The method to be adopted here is the same as that considered by Pal (1983), so to avoid mere repetition; we only write the final result. Hence, we finally obtain the solid displacement component on the free surface $z=-h$ of the soil layer as follows:

$$
v_{1}(x,-h, t)=L^{-1} I_{n}=\sum\left(I_{n 1}+I_{n 2}+I_{n 3}\right), \quad n=1,3,5, \ldots .
$$

where

$$
\begin{aligned}
& I_{n 1}= \begin{cases}\frac{2 P_{0}}{\pi} \int_{0}^{\infty} \eta^{\prime}(s) D_{n 1}(t, s) d s & \text { for } t \geq t_{1}^{(n)} \\
0 & \text { for } t \leq t_{1}^{(n)}\end{cases} \\
& I_{n 2}= \begin{cases}\frac{2 P_{0}}{\pi} f_{\theta n}^{(1)} \int_{0}^{\infty} \eta^{\prime}(s) D_{n 2}(t, s) d s & \text { if } \text { for } t_{12}^{(n)} \leq t \leq t_{1}^{(n)} \\
0 & \text { if for } t<t_{12}^{(n)}, \quad t>t_{1}^{(n)}\end{cases}
\end{aligned}
$$




$$
I_{n 3}= \begin{cases}\frac{2 P_{0}}{\pi} f_{\theta n}^{(2)} \int_{0}^{\infty} \eta^{\prime}(s) D_{n 3}(t, s) d s & \text { if } \text { for } t_{12}^{(n)} \leq t \leq t_{1}^{(n)} \\ 0 & \text { if for } t<t_{12}^{(n)}, \quad t>t_{1}^{(n)} .\end{cases}
$$

The following symbols here used:

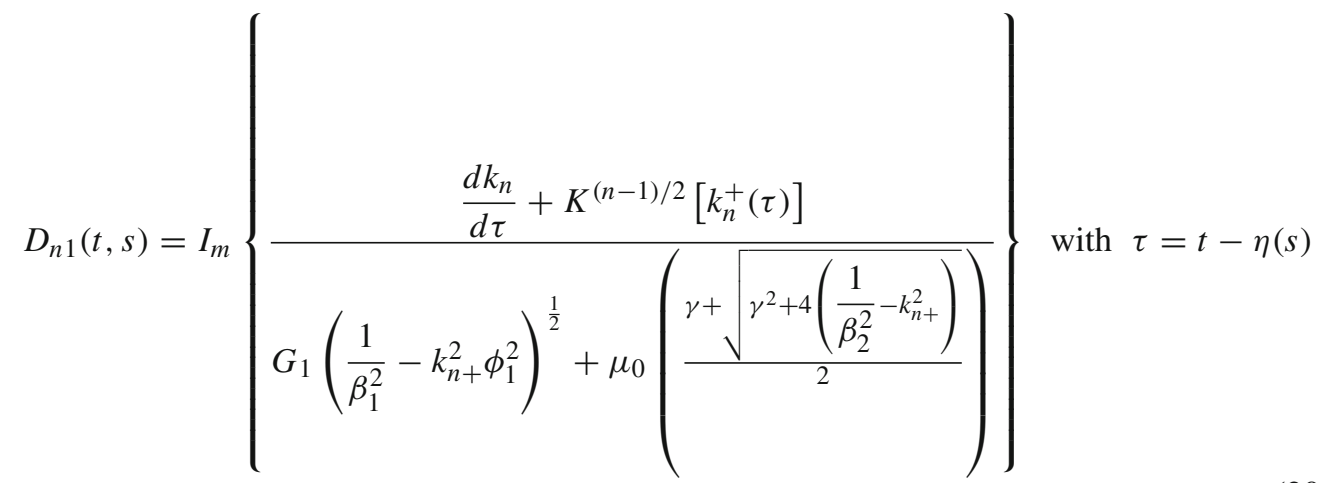

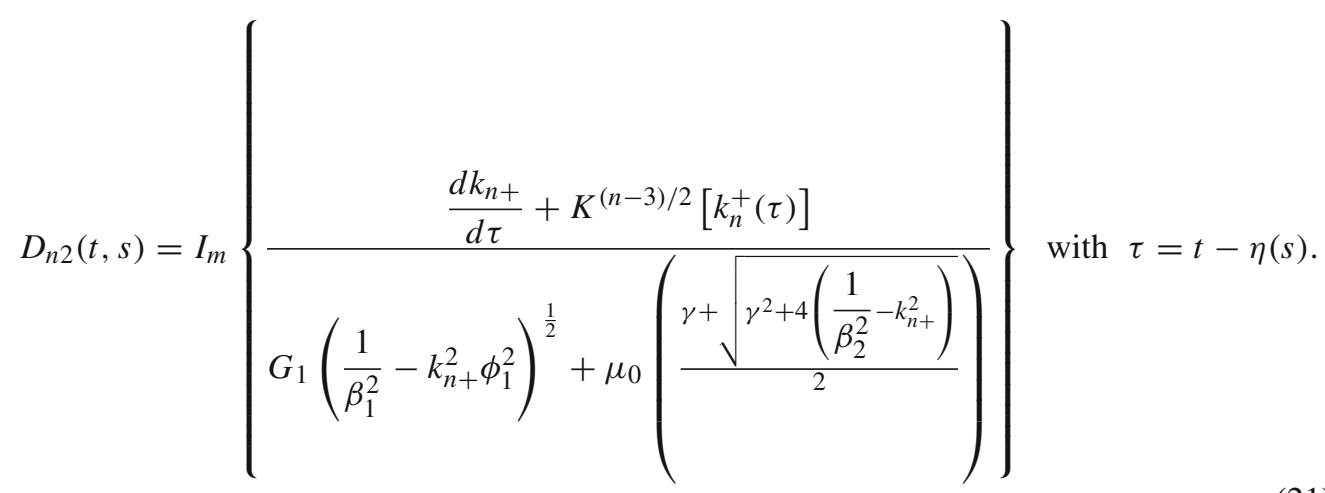

$D_{n 3}(t, s)$ is the same as $D_{n 2}(t, s)$ with $k_{n+}$ replaced by $k_{n-}$, as defined according to the equation

$$
k_{n \pm}=-\frac{\tau}{r_{n}} \cos \left(\theta_{n}\right) \pm \frac{i \sin \left(\theta_{n}\right)}{r_{n}}\left(\tau^{2}-\frac{r_{n}^{2}}{\phi_{1}^{2} \beta_{1}^{2}}\right)^{1 / 2},
$$

where $r_{n}^{2}=(x-s)^{2}+n^{2} h^{2}, \tan \left(\theta_{n}\right)=\frac{n h}{x-s}, 0 \leq \theta_{n} \leq \pi$, and

$$
k_{n}^{ \pm}=-\frac{\tau}{r_{n} \phi_{1}} \cos \left(\theta_{n}\right) \pm \frac{i \sin \left(\theta_{n}\right)}{r_{n} \phi_{1}}\left(\frac{r_{n}^{2}}{\phi_{1}^{2} \beta_{1}^{2}}-\tau^{2}\right)^{1 / 2}+i \xi
$$

where $0 \leq \theta_{n} \leq \cos ^{-1}\left(\frac{\beta_{2}}{\beta_{1}}\right)$ for $x>s$ and $\varepsilon \rightarrow 0$,

$$
f_{\theta n}^{(1)}= \begin{cases}1 & \text { for } \theta \leq \cos ^{-1}\left(\frac{\beta_{2}}{\beta_{1}}\right) \\ 0 & \text { elsewhere }\end{cases}
$$




$$
\begin{gathered}
f_{\theta n}^{(2)}=\left\{\begin{array}{ll}
1 & \text { for } \pi-\cos ^{-1}\left(\frac{\beta_{2}}{\beta_{1}}\right)<\theta_{n}<\pi \\
0 & \text { elsewhere }
\end{array},\right. \\
t_{n}^{(1)}=\eta(s)+\frac{r_{n}}{\beta_{1} \phi_{1}},
\end{gathered}
$$

and

$$
t_{12}^{(n)}=\eta(s)+\frac{r_{n} \cos \left(\theta_{n}\right)}{\beta_{2}}+r_{n} \sin \left(\theta_{n}\right)\left(\frac{1}{\phi_{1}^{2} \beta_{1}^{2}}-\frac{1}{\beta_{2}^{3}}\right)^{1 / 2} .
$$

The additional contour integration path (Cagniard) is shown in figure 2, where the hyperbola intersects branch cuts and the transformation from the $k$-plane to $\tau$-plane is defined by equation $b_{1} h-k x-k s=\tau$, where $\tau$ is real and positive.

\section{Brief discussion of the solution}

The term $I_{1}$ as defined in equation (19) is independent of $K$ and gives the contribution due to the direct wave from the source. The terms for different values of $n(n>1)$ in equation (19) gives the contributions due to the pulses or waves that suffer $n$ reflections at the interface of anisotropic soil layer and inhomogeneous medium. More explicitly, the terms $I_{n}$ (for all $n$ ) represent the integral sums of the disturbances due to all displacement discontinuities occurring at a given $x=0, z=-h$ and at any instant $t \geq 0$.

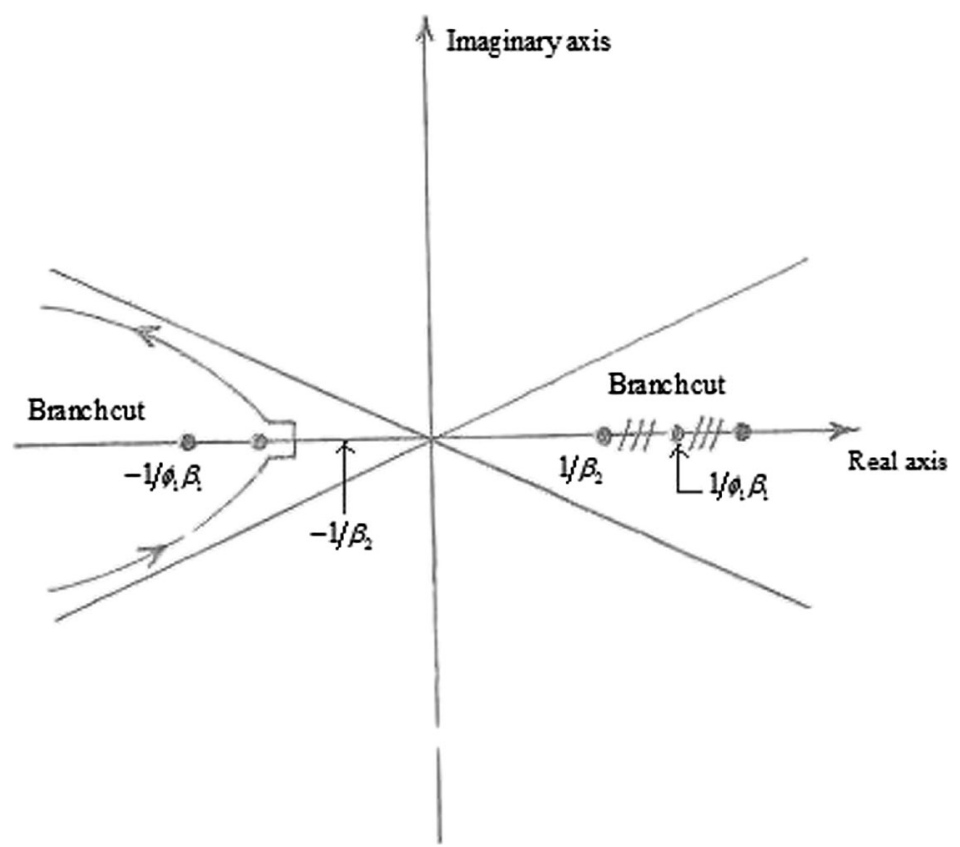

Figure 2. $\tau$-plane showing the contour integration. 


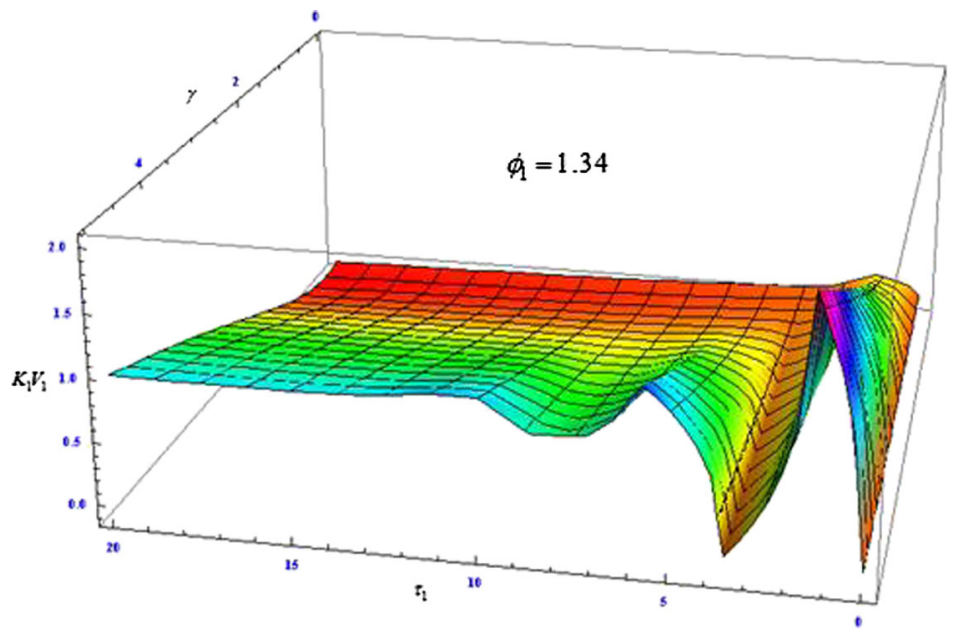

Figure 3. Variation of displacement $K_{1} V_{1}$ against $\tau_{1}$ and $\gamma$ for $\phi_{1}=1.34$ at $x=4 h$.

For the problem considered here, the shearing stress discontinuity generates wavelet at each point of the interface. A typical wavelet is emitted from the point $(s, 0)$ at the instant $\eta(s)$ and thereafter propagates with the velocity $\left(1 / \phi_{1} \beta_{1}\right)$. The radius of the shear wavelet at some later time is $[t-\eta(s)] \phi_{1} \beta_{1}$. The time at which a particular shear wavelet (for direct wave) arrives at the interface $(x,-h)$ is just $\eta(s)+\left[r_{1} /\left(\phi_{1} \beta_{1}\right)\right]$. Thus for any $t$ and any place $(x,-h)$, those points on the interface for which $t \geq \eta(s)+\left[r_{1} /\left(\phi_{1} \beta_{1}\right)\right]$ are undetected. Similar cases happen with a reflected $S$ wave. The above discussion justifies the condition imposed on $l(t)$, and consequently on $\eta(s)$, i.e., $\eta(s)$ is a monotonically increasing function of time $t$.

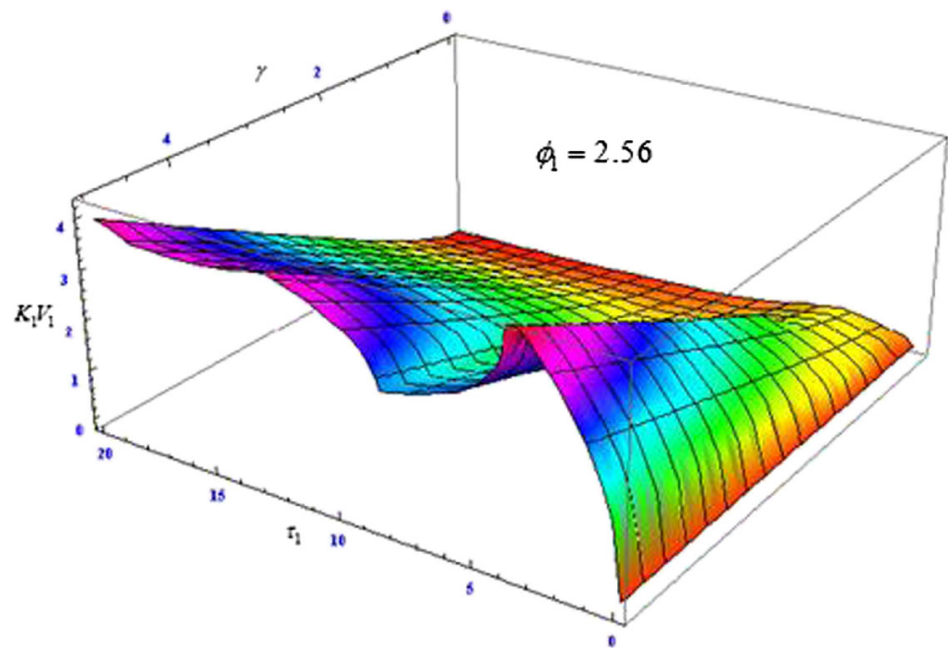

Figure 4. Variation of displacement $K_{1} V_{1}$ against $\tau_{1}$ and $\gamma$ for $\phi_{1}=2.56$ at $x=4 h$. 


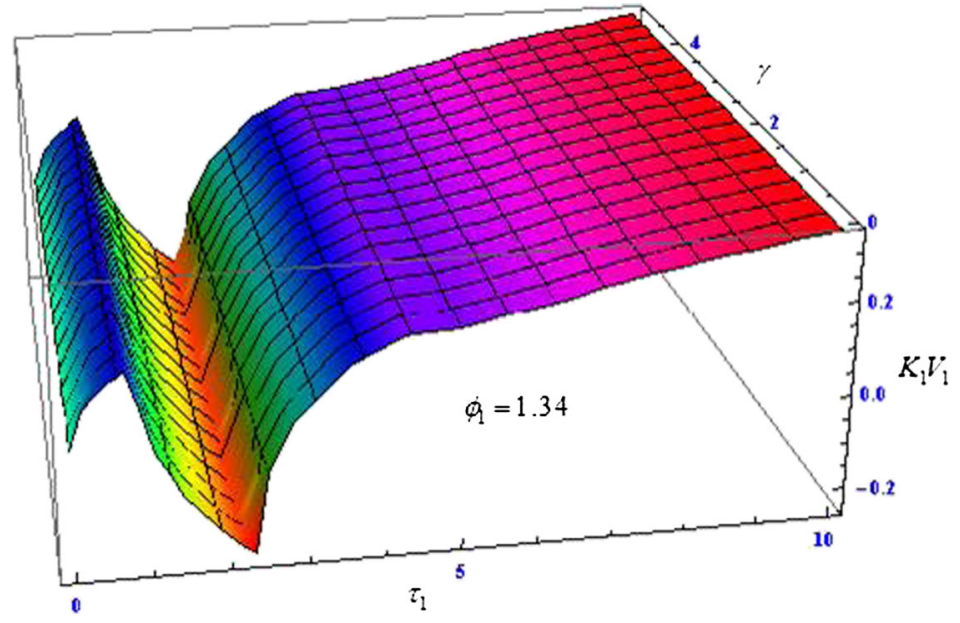

Figure 5. Variation of displacement $K_{1} V_{1}$ against $\tau_{1}$ and $\gamma$ for $\phi_{1}=1.34$ at $x=8 h$.

\section{Numerical results and discussion}

Consider a numerical example for a particular case of uniformly moving discontinuity.

We consider $f(x, t)=P_{0} h \delta(x-U t)$, which shows that stress discontinuity moves uniformly with constant velocity $U$ along $x$-axis. Hence in this case $\eta(s)=s / U$ and so $\eta^{\prime}(s)=1 / U$.

The values of alluvial soil $\left(\phi_{1}\right)$ are taken from Boer (2000) and correspond to incompressible binary model. The initial behaviour of the disturbance for few pulses is evaluated at a place $x=4 h$ and $8 h$ for alluvial soil $\phi_{1}=1.34$ and 2.56 as obtained by Pal \& Debnath (1979). According to this model, we have taken $\beta_{1}=2.54 \mathrm{~km} / \mathrm{s}, \beta_{2}=2.45 \mathrm{~km} / \mathrm{s}, \rho_{0} / \rho_{1}=0.91$ and $\beta_{1} / U=1.49$. The value of $K_{1} V_{1}(x,-h, t)$ is plotted against $\tau_{1}=\tau-\tau_{0}$, where $\tau_{0}$

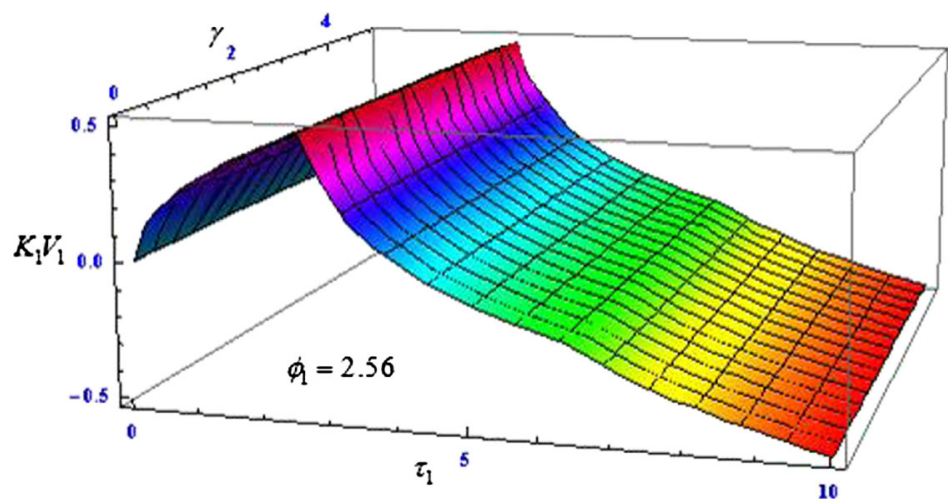

Figure 6. Variation of displacement $K_{1} V_{1}$ against $\tau_{1}$ and $\gamma$ for $\phi_{1}=2.56$ at $x=8 h$. 
denotes the time at which the disturbance arrives at the point of observation from the source to initial point with $K_{1}=\pi G_{1} / 2 P U$ and $\tau=t \beta_{1} / h$ (the non-dimensional time). The change occurs for a certain station $x=4 h$ and $8 h$ at different time for fixed values of alluvial soil at $\tau=\left(4^{2}+\phi_{1}^{2} n^{2}\right)^{1 / 2}$ and $\tau=\left(8^{2}+\phi_{1}^{2} n^{2}\right)^{1 / 2}, n=1,3,5, \ldots$. The values of $\tau_{0}$ for alluvial soil $\phi_{1}=1.34,2.56$ at $x=4 h$ are 4.21 and 4.74 and at $x=8 h$ are 8.11 and 8.39, respectively.

$$
\begin{gathered}
A^{0}\left(\theta_{n}\right)=\operatorname{Re} \frac{\left[\left(1-\phi_{1}^{2} \cos ^{2} \theta_{n}\right)^{\frac{1}{2}}-\frac{\rho_{0}}{\rho_{1}} \frac{\beta_{2}}{\beta_{1}}\left\{\frac{\gamma+\sqrt{\gamma^{2}+4\left(1-\frac{\beta_{2}^{2}}{\beta_{1}^{2}} \cos ^{2} \theta_{n}\right)}}{2}\right\}\right]^{n-1 / 2}}{\left[\left(1-\phi_{1}^{2} \cos ^{2} \theta_{n}\right)^{\frac{1}{2}}+\frac{\rho_{0}}{\rho_{1}} \frac{\beta_{2}}{\beta_{1}}\left\{\frac{\gamma+\sqrt{\gamma^{2}+4\left(1-\frac{\beta_{2}^{2}}{\beta_{1}^{2}} \cos ^{2} \theta_{n}\right)}}{2}\right\}\right]^{\frac{n-3}{2}}\left(\frac{\beta_{1}}{U}-\cos \theta_{n}\right)} . \\
n=1,3,5, \ldots \ldots \ldots . .
\end{gathered}
$$

$A^{0}\left(\theta_{n}\right)$ represents the reflection coefficient of SH type waves incident from the anisotropic soil layer to elastic inhomogeneous half-space.

Now when $x=4 h, t=\tau h / \beta_{1}$ for initial six values we have

$$
K_{1} V_{1}(x,-h, t) \approx\left[\begin{array}{l}
A^{0}\left(\theta_{1}\right) \cosh ^{-1}\left(\frac{\tau}{\sqrt{4^{2}+\phi_{1}^{2} \times 1^{2}}}\right) H\left(\tau-\sqrt{4^{2}+\phi_{1}^{2} \times 1^{2}}\right)+ \\
A^{0}\left(\theta_{3}\right) \cosh ^{-1}\left(\frac{\tau}{\sqrt{4^{2}+\phi_{1}^{2} \times 3^{2}}}\right) H\left(\tau-\sqrt{4^{2}+\phi_{1}^{2} \times 3^{2}}\right)+ \\
A^{0}\left(\theta_{5}\right) \cosh ^{-1}\left(\frac{\tau}{\sqrt{4^{2}+\phi_{1}^{2} \times 5^{2}}}\right) H\left(\tau-\sqrt{4^{2}+\phi_{1}^{2} \times 5^{2}}\right)+ \\
A^{0}\left(\theta_{7}\right) \cosh ^{-1}\left(\frac{\tau}{\sqrt{4^{2}+\phi_{1}^{2} \times 7^{2}}}\right) H\left(\tau-\sqrt{4^{2}+\phi_{1}^{2} \times 7^{2}}\right)+ \\
A^{0}\left(\theta_{9}\right) \cosh ^{-1}\left(\frac{\tau}{\sqrt{4^{2}+\phi_{1}^{2} \times 9^{2}}}\right) H\left(\tau-\sqrt{4^{2}+\phi_{1}^{2} \times 9^{2}}\right)+ \\
A^{0}\left(\theta_{11}\right) \cosh ^{-1}\left(\frac{\tau}{\sqrt{4^{2}+\phi_{1}^{2} \times 11^{2}}}\right) H\left(\tau-\sqrt{4^{2}+\phi_{1}^{2} \times 11^{2}}\right)
\end{array}\right] .
$$




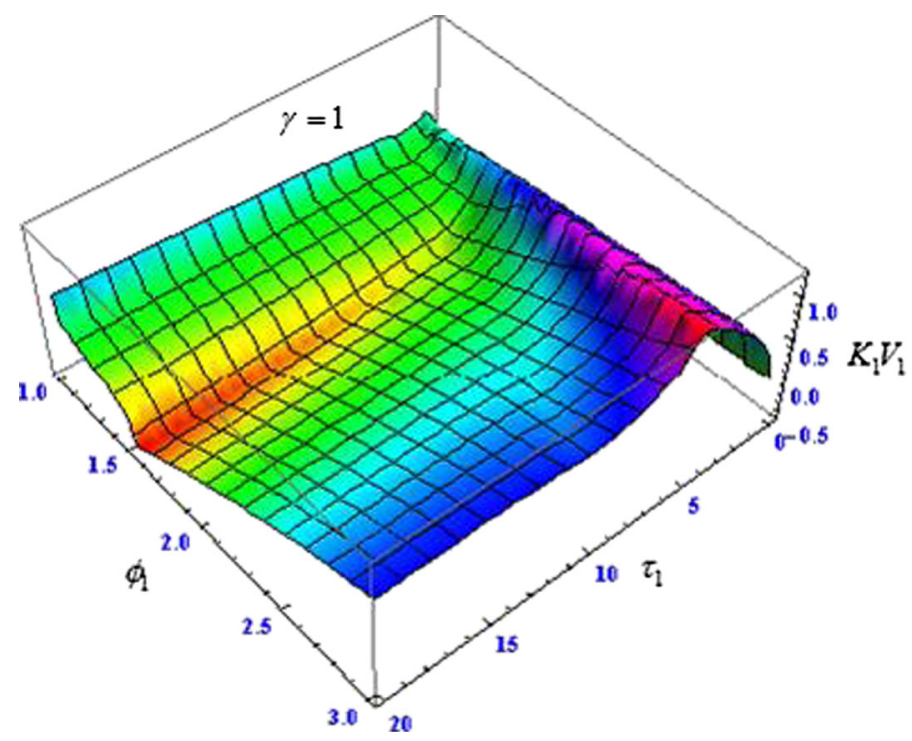

Figure 7. Variation of displacement $K_{1} V_{1}$ against $\tau_{1}$ and $\phi_{1}$ for $\gamma=1$ at $x=4 h$.

Now when $x=8 h, t=\tau h / \beta_{1}$ for initial six values we have

$$
K_{1} V_{1}(x,-h, t) \approx\left[\begin{array}{l}
A^{0}\left(\theta_{1}\right) \cosh ^{-1}\left(\frac{\tau}{\sqrt{8^{2}+\phi_{1}^{2} \times 1^{2}}}\right) H\left(\tau-\sqrt{8^{2}+\phi_{1}^{2} \times 1^{2}}\right)+ \\
A^{0}\left(\theta_{3}\right) \cosh ^{-1}\left(\frac{\tau}{\sqrt{8^{2}+\phi_{1}^{2} \times 3^{2}}}\right) H\left(\tau-\sqrt{8^{2}+\phi_{1}^{2} \times 3^{2}}\right)+ \\
A^{0}\left(\theta_{5}\right) \cosh ^{-1}\left(\frac{\tau}{\sqrt{8^{2}+\phi_{1}^{2} \times 5^{2}}}\right) H\left(\tau-\sqrt{8^{2}+\phi_{1}^{2} \times 5^{2}}\right)+ \\
A^{0}\left(\theta_{7}\right) \cosh ^{-1}\left(\frac{\tau}{\sqrt{8^{2}+\phi_{1}^{2} \times 7^{2}}}\right) H\left(\tau-\sqrt{8^{2}+\phi_{1}^{2} \times 7^{2}}\right)+ \\
A^{0}\left(\theta_{9}\right) \cosh ^{-1}\left(\frac{\tau}{\sqrt{8^{2}+\phi_{1}^{2} \times 9^{2}}}\right) H\left(\tau-\sqrt{8^{2}+\phi_{1}^{2} \times 9^{2}}\right)+ \\
A^{0}\left(\theta_{11}\right) \cosh ^{-1}\left(\frac{\tau}{\sqrt{8^{2}+\phi_{1}^{2} \times 11^{2}}}\right) H\left(\tau-\sqrt{8^{2}+\phi_{1}^{2} \times 11^{2}}\right)
\end{array}\right]
$$




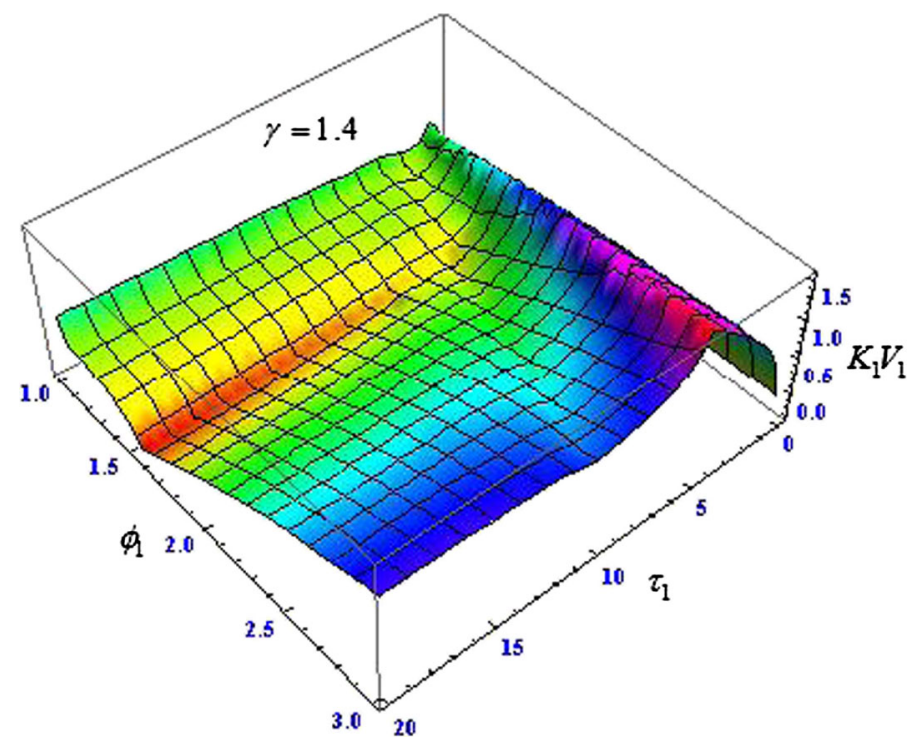

Figure 8. Variation of displacement $K_{1} V_{1}$ against $\tau_{1}$ and $\phi_{1}$ for $\gamma=1.4$ at $x=4 h$.

In order to see the effect of disturbance, we have considered the variation of $K_{1} V_{1}$ (i.e., displacement on the free surface) against the time of arrival of pulses (or the disturbance). The variations are considered in two cases. In first case, the varition are considered for fixed value of $\gamma$ (inhomogeneity parameter) and varying values of soil parameter $\phi_{1}$ for a fixed distances from the source i.e., $x=4 h$ and $8 h$. In-second case, the variations are considered for fixed value of soil parameter and varying values of $\gamma$ for the same fixed distances from the source i.e., $x=4 h$ and $8 h$. All these variation are shown as 3D-plots in figures 3-10. Figures 3-6 are drawn for variation of $K_{1} V_{1}$ with time for fixed value of soil parameter for the distances $x=4 h$ and $8 h$ whereas figures 7-10 are drawn for variation of $K_{1} V_{1}$ with time for fixed

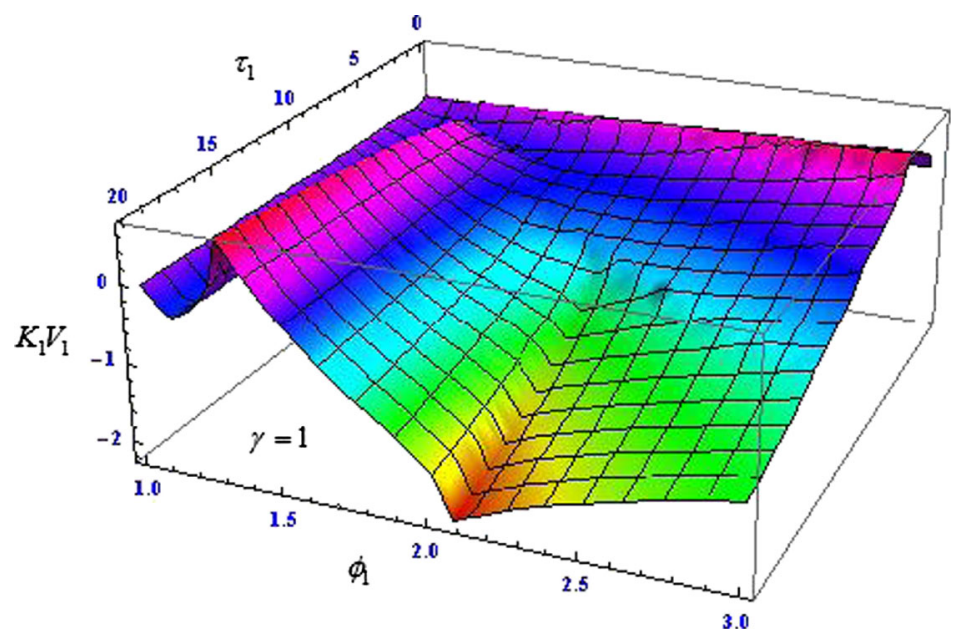

Figure 9. Variation of displacement $K_{1} V_{1}$ against $\tau_{1}$ and $\phi_{1}$ for $\gamma=1$ at $x=8 h$. 


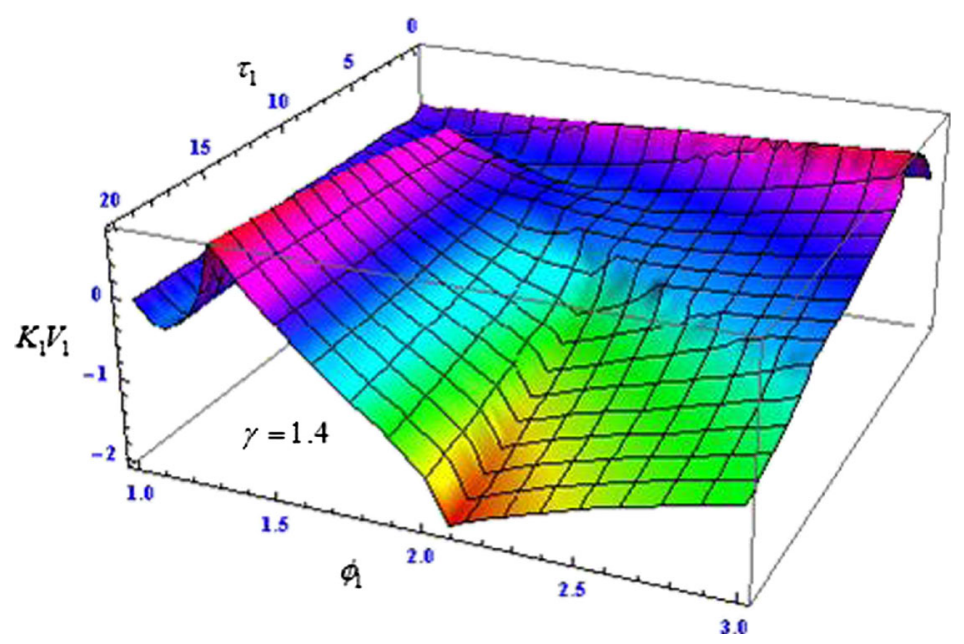

Figure 10. Variation of displacement $K_{1} V_{1}$ against $\tau_{1}$ and $\phi_{1}$ for $\gamma=1.4$ at $x=8 h$.

value of inhomogeneity parameter. It is observed from figures 3-6 that for both fixed distances $x=4 h$ and $x=8 h$ the discontinuity effects are pronounced and these are due to the arrival of pulses after repeated reflection and refraction from the source to the surface of the soil layer and back to interface. The same conclusion may be derived from figures 7-10. It is also concluded that as we increase inhomogeneity parameter (figures 3-6), the disturbance with discontinuity occurs very fast. From figures $7-10$, it is inferred that as we increase the soil parameter the disturbances are observed not very fast but slowly. Over all conclusion is that SH-type waves get disturbed when it crosses different layers. Two-dimensional curves are in well agreement with the curves (not surface plots) of Nag \& Pal (1977) (for isotropic case) and Pal \& Debnath (1979) (for anisotropic case).

\section{Conclusions}

The theoretical development of the dynamic properties for SH-waves using Cagniard-de Hoop technique in plane anisotropic soil layer overlying an inhomogeneous half-space is presented with an emphasis put on having the final formulae in a form that are readily programmable with Mathematica-7. A numerical discussion of a simple displacement time structure (surface plots) is given which shows the variation of inhomogeneity parameter and soil parameter on the disturbance of SH-waves. Surface plots give better visualization of stress discontinuity or jumps.

Finally, the shearing stress discontinuity is always associated with the propagation of cracks in earthquakes, the present study is having direct applications to Geophysics and Seismology. This problem serves as a convenient vehicle for introducing the analytical method, which may be applied to more complicated problems.

\section{Acknowledgements}

One of the authors, D M, is thankful to the Indian School of Mines (ISM), Dhanbad authorities and the University Grants Commission (UGC), New Delhi for Financial support in the form of a Research Fellowship. 


\section{References}

Brock L M 1982 The effect of displacement discontinuity derivatives on wave propagation-IV: Dislocation motion in a half space. Int. J. Eng. Sci, 20: 483-496

Cagniard L 1962 Reflection and refraction of progressive wave. Translated by Flinn and Dix. McGraw-Hill Book Company

Daley P F and Hron F 1977 Reflection and transmission coefficients for transversely isotropic media. Bull. Seis. Soc. Am. 67: 661-675

de Boer R 2000 Theory of Porous Media. Springer, Berlin

de Hoop A T 1960 A Modification of Cagniard's method for solving seismic pulse problem. Appl. Sci. Res. Sect. B, 8: 349-356

de Hoop A T 2002 Reflection and transmission of a transient, elastic, line-source excited SH wave by a planer, elastic bonding surface in solid. Int. J. Solid Struct. 39: 5379-5391

Freund L B 1972 Wave motion in an elastic solid due to a non-uniformly moving line load. Q. Appl. Math. 30: $271-281$

Garvin W W 1956 Exact transient solution of the buried line source problem. Proc. Royal Soc. A, 234: $528-541$

Mittal J P and Sidhu R S 1982 Generation of SH waves from a non-uniformly moving stress discontinuity in a layered half-space. Ind. J. Pure Appl. Math. 13: 682-695

Nag K R and Pal P C 1977 Disturbance of SH-type due to shearing stress discontinuity in a layered Halfspace. Geophys. Res. Bull. 15: 13-22

Pal P C 1983 Generation of SH-type waves due to non-uniformly moving stress-discontinuity in layered anisotropic elastic half-space. Acta Mech. 49: 209-220

Pal P C and Debnath L 1979 Generation of SH-type waves in a layered anisotropic elastic Media. Int. J. Math. Math. Sci. 2: 703-716

Romeo M 1997 SH surface waves in layered half-space. Q. J. Mech. Appl. Math. 50: 581-595

Sengupta P R and Nath S 2001 Surface waves in fibre-reinforced anisotropic elastic media. Sadhana 26: $363-370$

Tomar S K and Kaur J 2007 SH-waves at a corrugated interface between a dry sandy half-space and an anisotropic elastic half-space. Acta Mech. 190: 1-28 